\title{
Transcatheter solutions for transcatheter aortic valve replacement dysfunction: is redo transcatheter aortic valve replacement a durable option?
}

\author{
Andrew G. Chatfield, Anson Cheung, Mariama Akodad, Anthony Chuang, Laura Besola, \\ Stephanie Sellers, David A. Wood, Janarthanan Sathananthan, John Webb \\ Centre for Heart Valve Innovation, Centre for Cardiovascular Innovation, St. Paul's Hospital, University of British Columbia, Vancouver, BC, \\ Canada \\ Correspondence to: Anson Cheung. St. Paul's Hospital, 1081 Burrard Street, Vancouver, BC, V6Z 1Y6, Canada. Email: ACheung@providencehealth.bc.ca.
}

\begin{abstract}
As transcatheter aortic valve replacement (TAVR) expands into a younger and lower risk cohort of patients, many important clinical questions are raised, including the one of overall valve durability. Bioprosthetic valve dysfunction (BVD) is a complex clinical issue, of which structural valve deterioration (SVD) is a subcategory. Similar to surgical bioprosthesis, transcatheter heart valves (THVs) can fail over the years however, data on long-term THVs durability is lacking, especially in the lower risk cohort. Surgical explant with open aortic surgery or a second THV, described as redo-TAVR, are feasible options when the first THV fails. However long-term data in these patients is even more limited. Important clinical considerations such as the mechanism(s) of THV dysfunction, the type and timing of the second procedure must be carefully considered. There are also inherently important clinical concerns regarding redo-TAVR, such as coronary access and higher post procedure gradients. In the present keynote lecture, we review the diagnosis of THV dysfunction and transcatheter options available when SVD occurs.
\end{abstract}

Keywords: Aortic stenosis (AS); bioprosthetic valve dysfunction (BVD); coronary access (CA); redo transcatheter aortic valve replacement (redo TAVR); surgical aortic valve replacement (SAVR); structural valve deterioration/ dysfunction (SVD); transcatheter aortic valve replacement (TAVR); valve-in-valve (ViV)

Submitted Jun 17, 2021. Accepted for publication Aug 18, 2021.

doi: $10.21037 /$ acs-2021-tviv-85

View this article at: https://dx.doi.org/10.21037/acs-2021-tviv-85

\section{Introduction}

Transcatheter aortic valve replacement (TAVR) is a wellestablished treatment option for patients with severe aortic stenosis (AS) and prohibitive/high to intermediate risk for surgery (1-5). Recent trials have demonstrated either non-inferiority or superiority of TAVR compared with traditional surgical aortic valve replacement (SAVR) for younger/lower risk patients $(6,7)$. The expanding indications for TAVR means that by $2025,289,000$ TAVR procedures are projected to be performed annually across the globe (1). As this exponential growth in TAVR continues, it is paralleled by the importance of a thorough understanding of valve durability and the treatment options available when the transcatheter heart valve (THV) fails. Indeed, similarly to surgical bioprosthesis, THVs can fail over the years, particularly in a population of patients with a longer life expectancy. Initial TAVR trials included only a highly comorbid, elderly population and therefore, long-term THV durability was not a key early concern. However, with the expansion of TAVR into a younger, less comorbid population, the reality that the patient will outlive their first THV and require reintervention becomes a real concern. THV dysfunction will likely become a major cause of cardiovascular morbidity and possibly mortality in the future therefore, standardized definitions and early recognition to evaluate the THV durability is mandatory. Identifying patients with THV dysfunction early, with 
non-invasive tests and regular clinical follow-up, is best to allow treatment planning as required and ultimately, avoid catastrophic THV failure. Treatment options including surgical aortic valve replacement or a transcatheter approach, such as redo-TAVR, are dependent on the underlying mechanism of THV dysfunction, patient's anatomy, as well as individualized clinical situation and procedural risk.

In this review, we will discuss contemporary definitions of bioprosthetic valve dysfunction (BVD), current data on durability of surgical and THVs, evidence of different treatment strategies for BVD and implications for overall patient management.

\section{Defining THV dysfunction}

In order to try and standardize definitions of bioprosthetic valve durability, a consensus document from the European Association of Percutaneous Cardiovascular Interventions (EAPCI), the European Society of Cardiology (ESC), and the European Association of Cardiothoracic Surgery (EACTS) was produced in 2017 (8). In this consensus document, two types of structural valve deterioration (SVD) were described, namely hemodynamic and morphological SVD. Both types of SVD are assessed by echocardiography, hemodynamic SVD is due to permanent changes in valve function. Morphological SVD includes abnormalities in leaflet integrity and function. A patient-focused clinical endpoint called bioprosthetic valve failure (BVF) was added to incorporate clinical endpoints, such as reintervention or valve-related death. In 2018, Dvir et al. proposed a different definition of SVD focusing on the longitudinal deterioration of bioprosthetic valves, this document was important as it provided guidance on the timing of imaging during follow up (9). It must be stated that the overall driver for SVD is poorly understood and likely a complex process of which leaflet calcification appears to be an integral part (10). The Valve Academic Research Consortium (VARC) writing group recently released their VARC-3 update (11). This is the most up to date document regarding standardized definitions and clinical endpoints on BVF for TAVR and SAVR. See Figure 1 used with permission.

\section{The prognostic impact of THV dysfunction}

In the literature, SVD has been demonstrated to be associated with worsening morbidity and mortality and, a requirement for re-intervention. SVD can present in a variety of ways from subclinical changes only detected with surveillance echocardiography to a significant deterioration in clinical status or even, sudden cardiac death (12). Reoperation/intervention is recommended in symptomatic patients with a significant increase in trans prosthetic gradient or severe regurgitation (Class I, Level of Evidence C) and should be considered in asymptomatic patients with significant THV dysfunction, provided they remain at lowsurgical risk (Class IIa, Level of Evidence C) (13) (Figure 2).

\section{Imaging for BVD}

The Valve Academic Research Consortium-3 (VARC-3) consensus document suggests echocardiography as the principal imaging modality for assessment of THV function immediately before initial hospital discharge, at six months, one year and annually, thereafter (11). Transcatheter valve stenosis and regurgitation can be assessed using a number of different imaging modalities and are associated with worse clinical outcomes if they are present to varying degrees $(14,15)$. See Figure 3 for examples for SVD and non-SVD.

Multidetector computed tomography (MDCT) is another complementary tool in the non-invasive work up of suspected valve degeneration and pre-procedural planning. MDCT offers excellent spatial resolution and is very sensitive at detecting hypoattenuating leaflet thickening (HALT), leaflet calcification, pannus as well as endocarditis (see Figure 4A-4D). MDCT may also be useful to assess the mechanism of THV dysfunction including malposition or under expansion. Currently, MDCT is unable to provide hemodynamic data and is therefore, of reduced utility for the diagnosis of SVD $(16,17)$.

\section{Durability of SAVR}

The long-term outcomes and importance of SVD were analysed in a contemporary cohort of all-comers undergoing SAVR (18). In 672 consecutive patients with a mean age of 72 undergoing SAVR with a bioprosthetic implant from 2002-2004, echocardiographic and outcome data was collected on 624 (93\%) of the patients at discharge and 209 patients at ten years follow up (87\% of the patients at risk). SVD was defined as subclinical if there was an increase $>10 \mathrm{mmHg}$ in mean aortic valve gradient and decreased $>0.3 \mathrm{~cm}^{2}$ in valve area and/or new-onset mild or moderate aortic regurgitation (AR). SVD was clinically relevant if there was an increase $>20 \mathrm{mmHg}$ in mean aortic gradient and valve area decreased $>0.6 \mathrm{~cm}^{2}$ and/or new-onset moderate- 

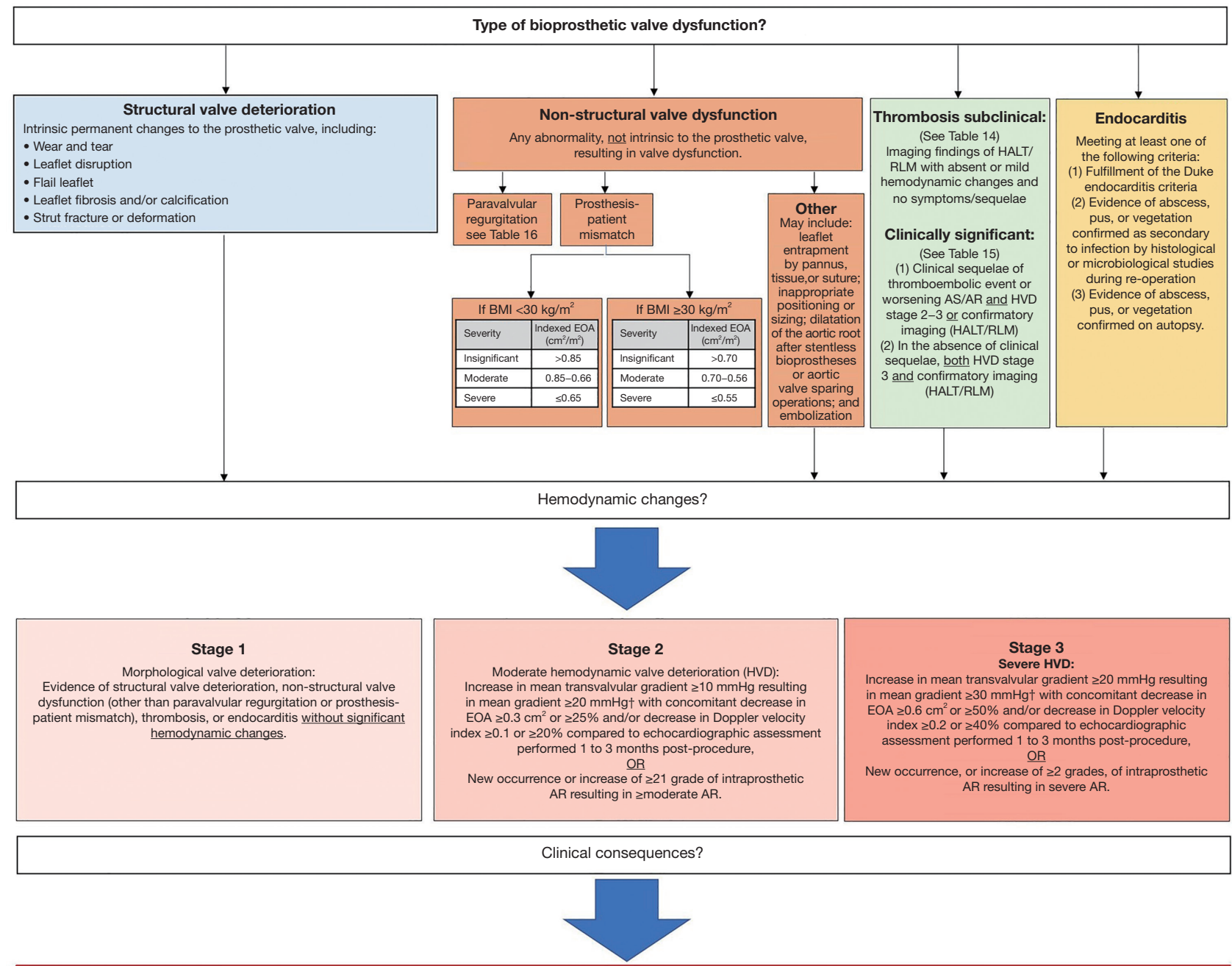

Bioprosthetic valve failure (BVF)

Stage 1: Any bioprosthetic valve dy
Stage 2: Aortic valve reintervention

Stage 3: Valve-related death

B

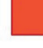

Figure 1 Bioprosthetic valve dysfunction and bioprosthetic valve failure.

to-severe AR. At ten years follow up, 423 (64.3\%) of the patients had died. Predictors of SVD included a certain aortic bio-prosthesis and a large body mass index. The incidence of clinically relevant SVD was $6.6 \%$, and $30.1 \%$ had subclinical SVD. Of those with clinically relevant SVD, $83 \%$ underwent aortic reintervention with nearly half (44\%) having a THV placed into the degenerated surgical valve, also described as a 'Valve-in-Valve' (ViV) TAVR procedure. This original study demonstrated a relatively high rate of subclinical SVD, but a low rate of clinically relevant SVD.

\section{THV durability}

Since the first TAVR valve was implanted in 2002 (19), ongoing refinements to the procedure and complex engineering of the THV's has meant the TAVR procedure has continued to evolve. A report from the PARTNER I trial showed comparable echocardiographic parameters for the balloon expandable SAPIEN valve (Edwards Lifesciences, Irvine, CA, USA) and SAVR bioprosthesis in patients who remained alive at five years (20). Of those 

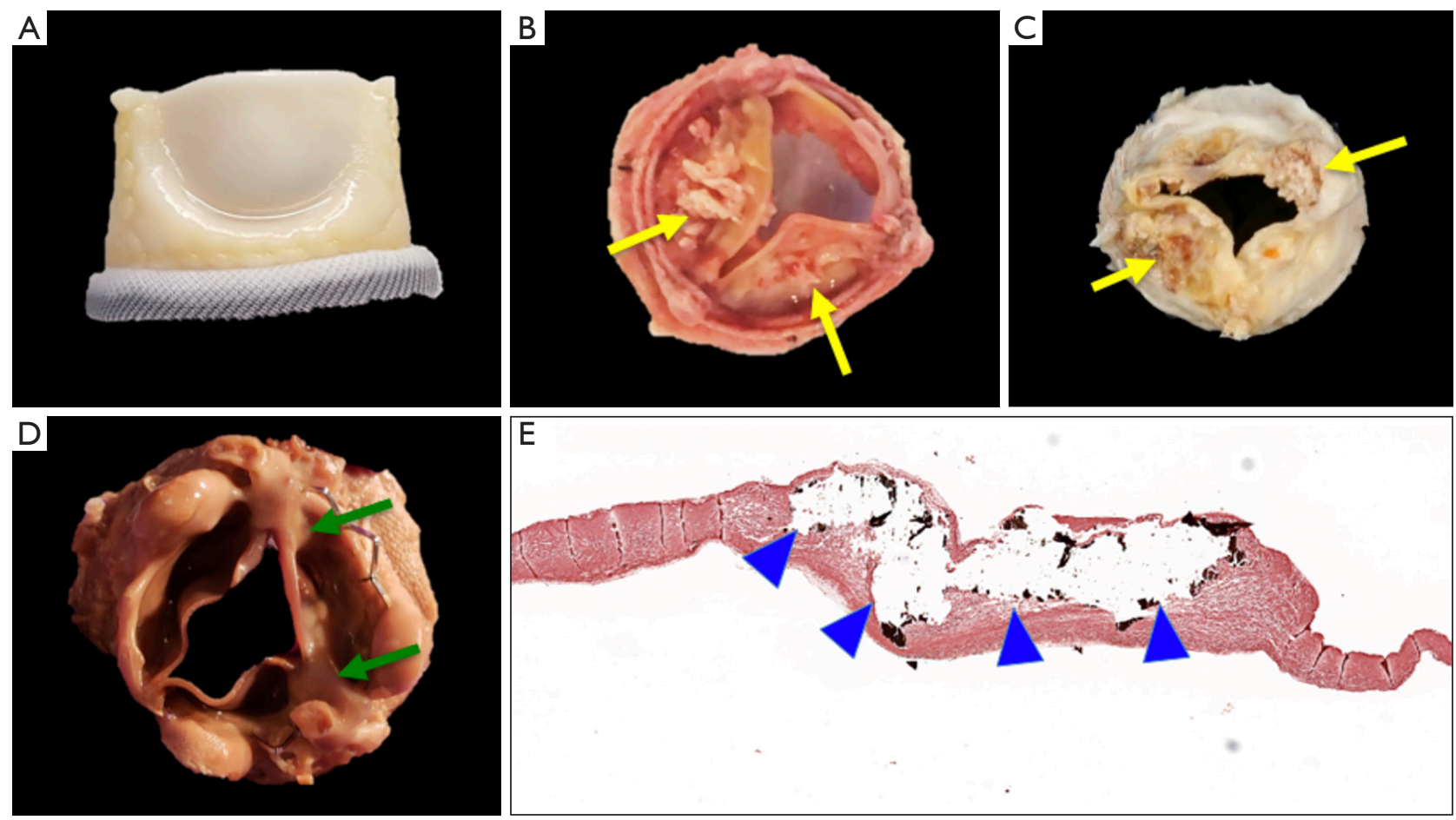

Figure 2 Explanted gross and histologic bioprosthetic valve pathology. (A) New (non-implanted) 25 mm MitroFlow showing pristine pericardial leaflets; (B) highly calcified explanted $27 \mathrm{~mm}$ MagnaEase (yellow arrows show calcific nodules; (C) highly calcified explanted surgical bioprosthetic valve explant; (D) highly fibrotic valve-in-valve (26 mm SAPIEN in 27 mm Mosaic) (green arrows show fibrosis restricting leaflets); (E) histological image showing calcification in the leaflet of a bioprosthetic valve as shown by Von Kossa staining (blue arrow heads).

who were alive at five years, 86 TAVR and 48 SAVR patients had their post implant echocardiogram; and fiveyear echocardiographic results matched using a paired $t$-test. Those that had TAVR had stable AV mean gradient post implant at five years, $11.5 \pm 5.4$ and $11.0 \pm 6.3 \mathrm{mmHg}$ $(\mathrm{P}=0.41)$, respectively. Long-term evaluation of the SAVR cohort showed similar trends and there was a low rate of adverse events in those still alive in both groups. Analysis of five-year data is similar when looking at the self-expanding valve CoreValve (Medtronic Inc., Minneapolis, Minnesota) showing a low rate of BVD at 1.4\% (21). Only patients that had consecutive five-year follow-up data were included (353 patients) from June 2007-August 2009. All outcomes were reported as per the then current VARC (Valve Academic Research Consortium)-1 criteria. With regard to echocardiographic follow-up, the mean aortic valve gradient at five years was $12.8 \pm 10.9 \mathrm{mmHg}$ in the TAVR group. Late BVD was reported in five $(1.4 \%)$ cases, two $(0.56 \%)$ patients had redo TAVR for restenosis and the remaining three had no further intervention. Ten $(2.8 \%)$ patients had late mild stenosis with a mean transaortic gradient $\geq 20 \mathrm{mmHg}$. No other cases of SVD or non-SVD were observed, late valve embolization and valve thrombosis were unfortunately not reported. Overall, it was shown the use of the self-expanding CoreValve (Medtronic Inc., Minneapolis, Minnesota) for severe AS in a high-risk population has durable results out to five years in those patients who remain alive. In the Nordic Aortic Valve Intervention (NOTION) trial (22), all comers with severe AS and low surgical risk were randomized 1:1 to TAVR [139] using the selfexpanding CoreValve (Medtronic, Minneapolis, Minnesota) or SAVR [135]. Moderate/severe SVD was defined as a mean gradient $\geq 20 \mathrm{mmHg}$, an increase in mean gradient $\geq 10 \mathrm{mmHg}$ three months post-procedure, or more than mild intra-prosthetic valve leak either new or progressive from three months post-procedure. Non-SVD was defined as moderate/severe patient-prosthesis mismatch at three months or moderate/severe paravalvular leakage. BVF was 

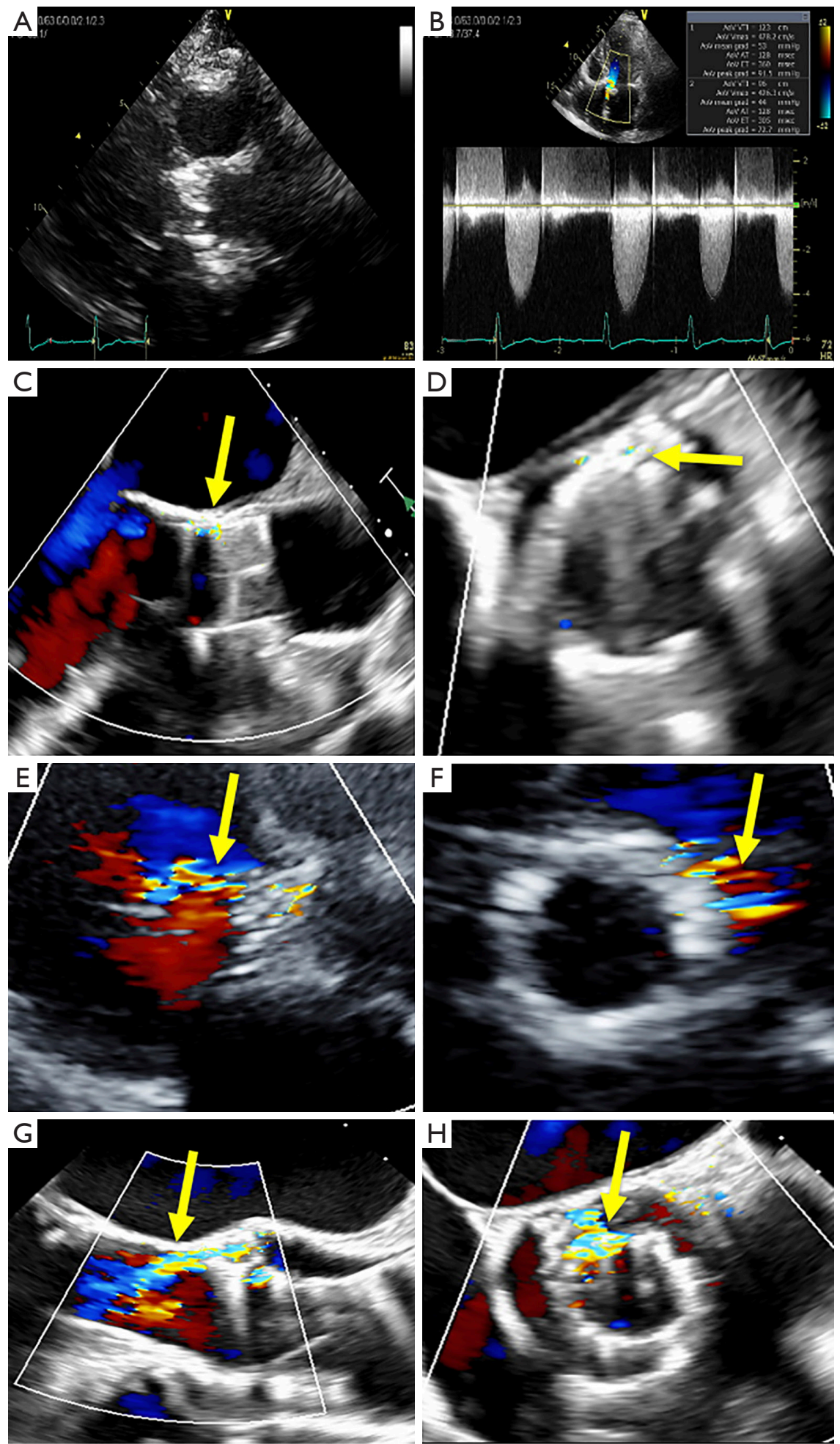

Figure 3 (A,B) TTE demonstrating a SAPIEN 3 in the aortic position with calcification of THV leaflets (A) with high post implant gradient at one year (B), consistent with hemodynamic SVD. TEE demonstrating mild, moderate and severe PVL on long axis and short axis imaging consistent with non-SVD (C-H) (yellow arrows depict location leak). TTE, transthoracic echocardiography; THV, transcatheter heart valve; SVD, structural valve deterioration; TEE, transoesophageal echocardiography; PVL, paravalvular leak. 

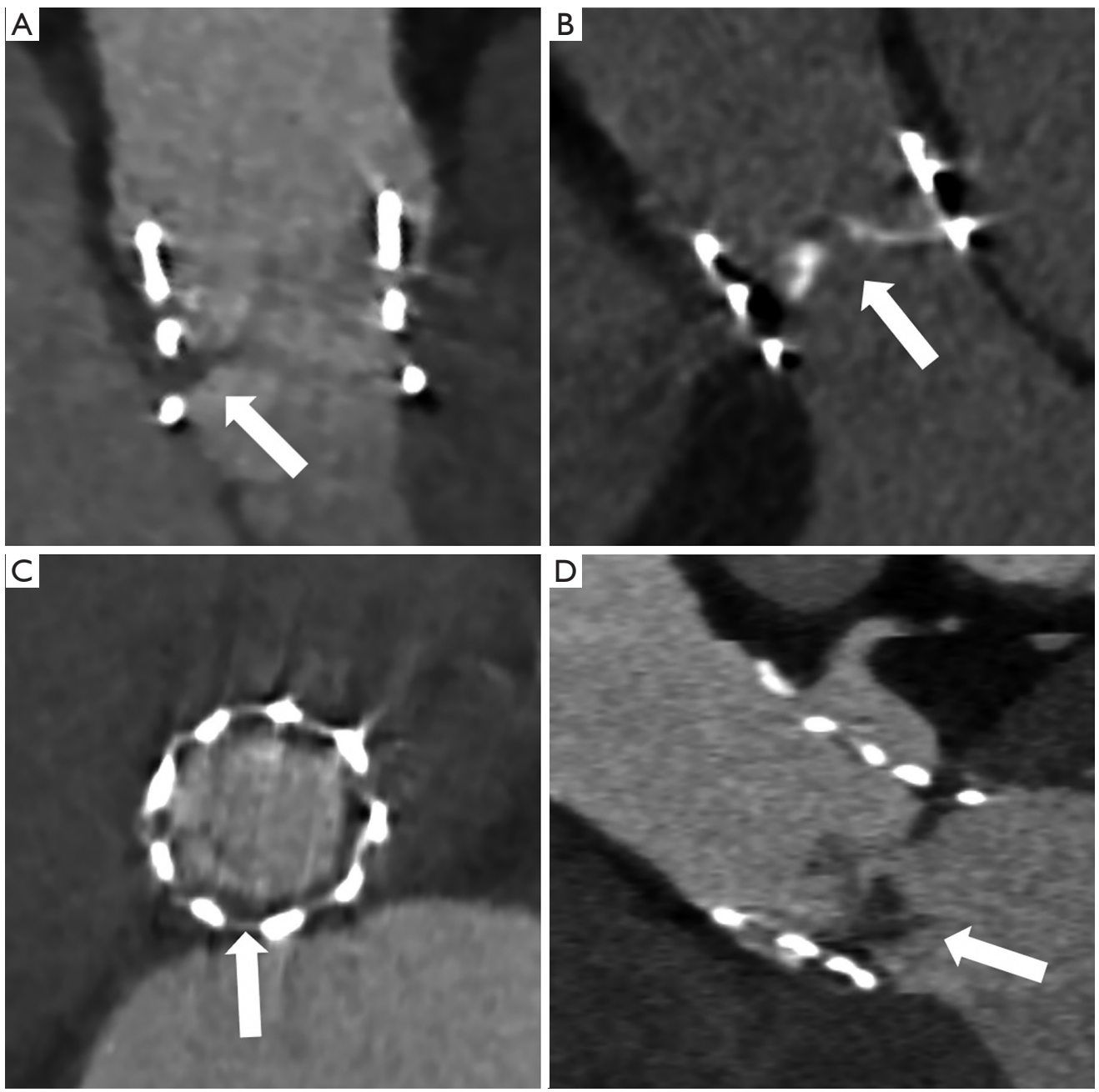

Figure 4 Computed tomography demonstrating structural and non-structural valve degeneration. (A) HALT on the aortic surface of a 23 mm Sapien THV (white arrow). (B) Calcification of Sapien $23 \mathrm{~mm}$ THV leaflets seven years post implant (white arrow). (C) Circumferential pannus at the inflow of a Sapien XT $23 \mathrm{~mm}$ (white arrow). (D) Hypoattenuating tissue adherent to the ventricular surface of the THV leaflet consistent with possible endocarditis (white arrow). THV, transcatheter heart valve; HALT, hypoattenuating leaflet thickening.

defined as valve-related death, aortic reintervention or severe hemodynamic SVD. At six years follow-up, SVD was higher in the SAVR group compared with TAVR (24\% vs. $4.8 \% ; \mathrm{P}<0.001)$, there were no differences in non-SVD (58\% vs. $54 \% ; \mathrm{P}=0.052)$ or endocarditis $(5.9 \%$ vs. $5.8 \%$; $\mathrm{P}=0.95)$. BVF was similar in SAVR and TAVR at six years (6.7\% vs. $7.5 \% ; \mathrm{P}=0.89$ ).

The FRANCE-2 registry (FRench Aortic National CoreValve and Edwards) is the largest high-surgical risk registry to date (23). This multicentre prospective registry of 4,201 patients with severe AS felt to be at prohibitive risk for SAVR were treated with TAVR across thirty-four sites in France and Monaco from Jan 2010-Jan 2012. Follow up consisted of regular echocardiographic surveillance and clinical review. All-cause mortality was high at five years at $60.8 \%$, reflecting this high-risk population. Echocardiographic follow-up data was available in 459 patients at the five-year follow-up. There was little change in the aortic valve mean gradients over the five years, with a mean change of $3 \mathrm{mmHg}$ (9 to $12 \mathrm{mmHg}$ ) (23). Moderate SVD occurred in $6.9 \%$ at one year and $12.4 \%$ at four to five years of follow-up. Severe SVD $(n=60)$ was present in only $1.4 \%$ at one year, increasing to $2.9 \%$ between four and five years. There was no clinically 
significant difference between THV platforms (balloon expandable versus self-expanding) in the rates of SVD $(\mathrm{P}=0.756)$. This data does show favourable valve durability with only $2.9 \%$ of the surviving patients at five years being diagnosed with severe SVD. It was observed that smaller valve sizes were associated with higher rates of SVD. Unfortunately, no data were presented on the rates of reintervention for BVF.

\section{Durability of TAV-in-SAV (ViV) as an alternative to repeat SAVR}

If the SAVR valve fails, the possibility of THV into the SAVR valve $(\mathrm{ViV})$ is a well-established treatment option in selected patients (24). There is reassuring mediumterm evidence to show that $\mathrm{ViV}$ is safe and as reliable as re-do SAVR, however, long-term results are not yet available. Registry data has shown that in certain highrisk patients ViV with a balloon expandable device for BVF is a safe procedure with low mortality, improved valve hemodynamics and overall patient quality of life at thirtyday and one-year follow-up (24). Patients with symptomatic degeneration of surgically implanted bioprosthetic valve were prospectively enrolled in the multicentre PARTNER (Placement of Aortic Transcatheter Valves) $2 \mathrm{ViV}$ trial and continued access registries. ViV procedures were performed in 365 patients (96 in the initial registry and

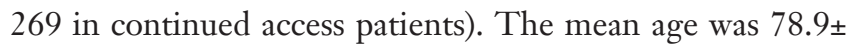
10.2 years and the Society of Thoracic Surgeons Predicted Risk of Mortality score (STS) was $9.1 \% \pm 4.7 \%$. Adverse events were low at thirty-days, with all-cause mortality at $2.7 \%$, stroke $2.7 \%$, major vascular complication rate of $4.1 \%$, conversion to surgery $0.6 \%$ and coronary occlusion only $0.8 \%$. At one-year, all-cause mortality was $12.4 \%$ and this mortality rate was notably lower in the continued access registry indicating a learning curve with the procedure. At the one-year mark, only $1.9 \%$ of the ViV TAVR patients had greater than mild paravalvular regurgitation and the mean gradient (MG) across the aortic prosthesis was $17.6 \mathrm{mmHg}$.

Similar findings were seen with The CoreValve U.S. Expanded Use Study in patients with BVF at prohibitive risk for redo surgery (25). The CoreValve U.S. Expanded Use Study enrolled 233 patients with symptomatic BVF unfit for redo surgery. Thirty-day and one-year outcomes were analysed, BVF was due to stenosis in $56 \%$, regurgitation in $22 \%$ or a combination in $22 \%$. A total of 227 patients underwent attempted $\mathrm{ViV}$ which was successful in 225 (99.1\%). Patients were elderly $(76.7 \pm 10.8$ years), STS of $9 \% \pm 6.7 \%$ and were highly symptomatic with $\geq 86 \%$ having New York Heart Association functional class $\geq$ III. Moderate AR occurred in $3.5 \%$ of patients at thirty days and $7.4 \%$ at one year. The mean aortic valve gradient was $17 \pm 8.8 \mathrm{mmHg}$ at thirty days and $16.6 \pm 8.9 \mathrm{mmHg}$ at one year. Overall, as mentioned there was significantly improved valve hemodynamics, and greatly improved quality of life and low rates of moderate AR.

Further data from a nationwide study from France has shown the ViV compared with redo SAVR has more favourable results in the short term but there were no observed differences in major cardiovascular outcomes during long term follow up (26). From 2010-2019, registry data was collected in France of 4,327 patients who had $\mathrm{ViV}$ or redo SAVR for failed aortic bioprosthesis. After propensity matching, 717 patients were analysed in each $\mathrm{arm}$. At thirty days, $\mathrm{ViV}$ had lower rates of the composite of all-cause mortality, all cause stroke, myocardial infarction (MI) and major or life-threatening bleeding (OR 0.62; $0.44-0.88, \mathrm{P}=0.03$ ). Median follow up was 516 days there was no difference in the composite endpoint of the same variables as well as rehospitalization for heart failure (OR $1.18 ; 0.99-1.41 ; \mathrm{P}=0.26)$. If rehospitalization for heart failure and pacemaker rates were analysed as separate outcomes, they were more frequent in the $\mathrm{ViV}$ group (26). Lastly, a recent meta-analysis comparing the outcomes of ViV vs. redo SAVR for failed bioprosthetic aortic valves demonstrated a similar risk of all-cause mortality, cardiovascular mortality, MI, permanent pacemaker implantation and $\geq$ moderate paravalvular leak (OR 1.15; 95\% CI: 0.93 to $1.43 ; \mathrm{P}=0.21),(27)$.

\section{Durability of redo-TAVR}

Redo-TAVR for patients with a failing THV may become an established treatment option in the future. Currently there is limited long-term data in patients who have undergone redo-TAVR. In the Redo-TAVR registry, data was collected from thirty-seven TAVR centres to evaluate safety and efficacy of redo-TAVR (28). The patients in the registry were classified as either probable TAVR failure if they presented within one year of the implant or probable THV failure if they presented after one year of their index TAVR procedure. There was a large number of TAVR implants totalling 63,876, of which $212(0.33 \%)$ were redo-TAVR procedures. Seventy-four implants were within one year of the index event and the majority of 138 
implants were completed $\geq 1$ year after the index TAVR. Of the probable THV failure group the mean time of TAVR to redo-TAVR was five years (three to six years). If the redo-TAVR was done within the first year (probable TAVR failure) the main reason was due to regurgitation $(\mathrm{n}=54,72.9 \%)$. When the redo-TAVR was completed after one year (probable THV failure) the majority were due to AS ( $\mathrm{n}=51,37 \%)$ followed by a combination of AS/ $\operatorname{AR}(n=45,32.6 \%)$ and lastly isolated AR $(n=41,29.7 \%)$. The implantation of the second THV was successful in 180 patients $(85.1 \%)$ as per the previous VARC-2 criteria, with high residual gradients being the most common cause for failure. Importantly at thirty-day and one-year follow up residual gradients were stable at $12.6 \pm 7.5$ and $12.9 \pm 9.0 \mathrm{mmHg}$, respectively. In terms of risk to the patients, the complication rate of redo-TAVR was low with three strokes, two coronary obstructions and seven device malpositions. Twenty new permanent pacemakers were required. The was no mortality related to the procedure and importantly there was a significant improvement in patient quality of life. It is important to mention that this is a multicentre registry and no information was given to the number of patients declined for redo-TAVR by their respective heart teams, i.e., this was a highly selected group of redo-TAVR patients and caution should be taken when interpreting the rates of complications.

This is the largest registry to date for redo-TAVR in failing THV valves. Survival at thirty days and at one year was high in both groups (probable TAVR and THV failure). The rates of survival at thirty days were $94.6 \%$ and $98.5 \%$ $(\mathrm{P}=0.101)$ and $83.6 \%$ and $88.3 \%(\mathrm{P}=0.335)$ at one year for patients presenting with early and late valve dysfunction, respectively (28). It would appear from the registry data that this is a safe and effective option for a selected cohort of patients with both late and early TAVR dysfunction.

\section{Bench testing-a way to explore valve durability and feasibility}

The data from Landes et al. (28), provides excellent insights into the potential for redo-TAVR in patients with failed $\mathrm{THV}$, however, once again long-term follow up is not yet available. In an attempt to explore this further, bench testing has become a novel way to try and understand the durability of THVs and some of the technical aspects involved with redo-TAVR. An early study explored the durability of both nominally and non-nominally deployed balloon expandable THVs to one billion cycles (approximately twenty-five years) with accelerated wear testing compared with Magna Ease surgical valves (29). The results showed very small percentages of THVs having a significant regurgitant fraction, demonstrating excellent THV durability to the equivalent of twenty-five years of wear. A recently published bench study again by Dr. Sathananthan et al. (30), assessed the safety and feasibility of various THV platforms, sizes and implant locations in the setting of redo-TAVR. The authors found that the majority of THV combinations they studied demonstrated favourable hydrodynamic performance. As expected, the first THV implant (design and size) is vitally important when considering the design, implant location, size and hemodynamic implications of the second THV (30).

\section{Sequence of valve intervention in younger patients}

The randomized trials of TAVR as described above initially started with patients that were not eligible for surgery and in-fact TAVR was a last resort. Within the last ten years the trial data has shown that TAVR is non-inferior to surgery. With a more patient centred approach in medicine patients are frequently wanting TAVR rather than surgery due to its less invasive nature and quicker recovery time. As younger patients ( $\leq$ seventy-five years old) are being discussed for aortic valve replacement, all potential options need to be considered. Pasala et al. (31), suggested that a 'paradigm shift' in the treatment algorithm for younger patients needing aortic valve intervention would be required in the future with the changing landscape of transcatheter option and indications. They propose that younger patients in discussion with the heart team first have TAVR, the TAVR may last ten years taking the patients though to age appx sixty to eighty years of age at which point SAVR and then if the SAVR fails the patient may be eligible for ViV TAVR in their later years i.e., >eighty years of age (Figure 5). This intervention algorithm would negate the need for long term anticoagulation and the number of open-heart procedures would be kept to a minimum. Some patients requiring aortic intervention will continue to require SAVR due to an indication for other conditions such as coronary artery disease, root enlargement or concomitant valve disease.

An important point to note in above proposed algorithm is some of the inherent difficulties of surgical explant of a failed TAVR valve. A large analysis on TAVR explant using registry data from the Society of Thoracic Surgeons national database was reported in 2020 (32). The registry 


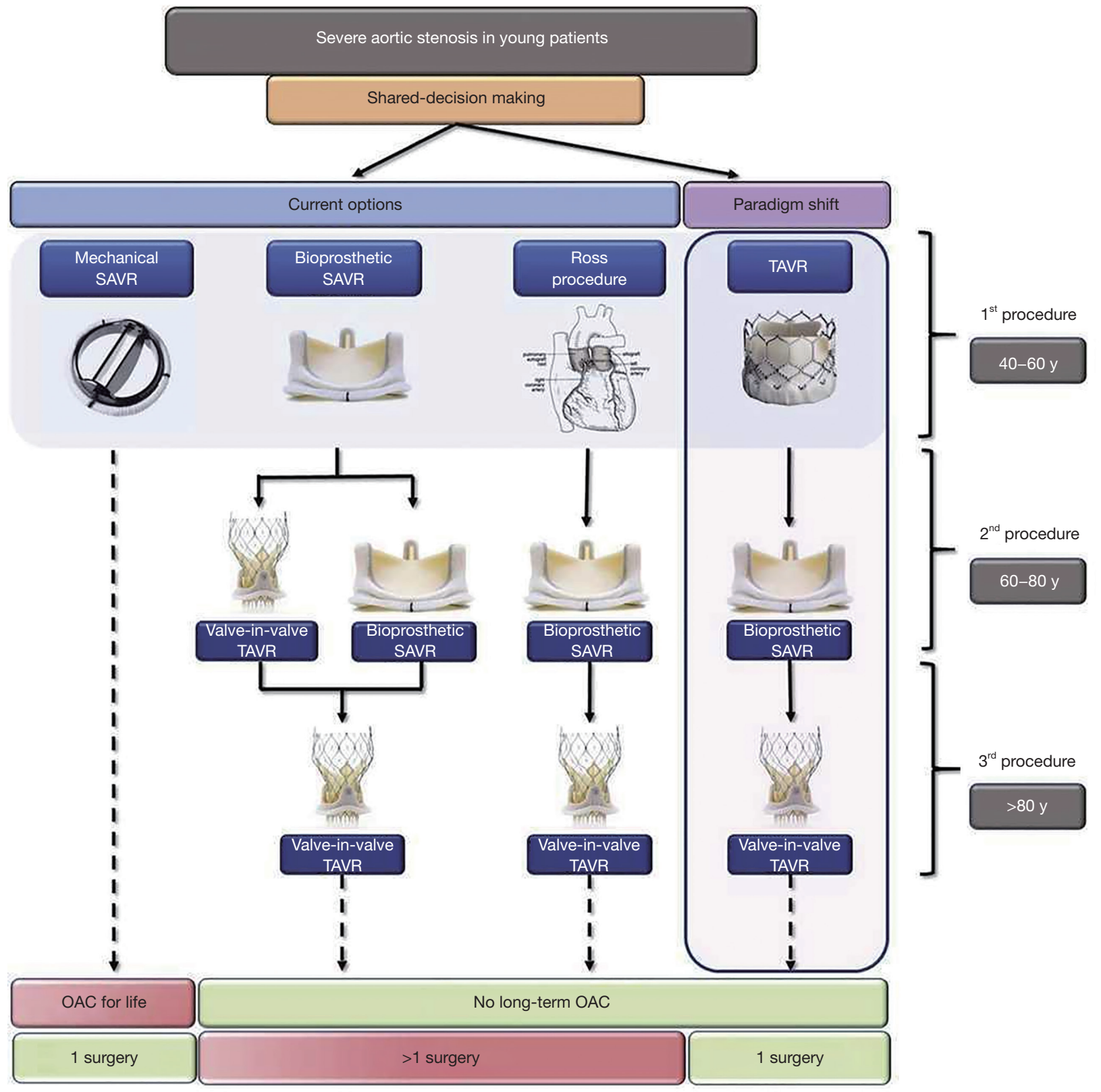

Rev Esp Cardiol. 2018;71:141-5

Figure 5 A potential treatment options of the long-term management of aortic stenosis (used with permission).

included all patients with a previous TAVR and then underwent SAVR anytime at least one day after the TAVR valve from 313 centres. From 2011-2018, 782 patients with TAVR explant were identified. The median age was seventy-four [interquartile range (IQR), 67-81]. 39\% were female and $55 \%$ had $\geq$ NYHA III symptoms. The majority $(\mathrm{n}=437,55 \%)$ underwent a concomitant procedure at the time of TAVR explant, aortic repair was the most common $(n=200 ; 46 \%)$. The main reason of THV failure and therefore indication for TAVR explant was due to a failed repair/positioning/sizing issue (27\%), followed next by AR or paravalvular leak (22\%), stenosis (20\%), endocarditis (18\%), SVD (7\%) and remained uncertain in $7 \%$. There was a high overall thirty-day mortality rate at $19.4 \%$, 
isolated SAVR patients had an STS score of $13.1 \%$. Overall, this is the largest registry of patients who underwent TAVR explant with subsequent SAVR. There are limitations interpreting the number of explants relative to the actual number of TAVR implants and the exact time from THV implant to explant in a large number of cases (32). It was noted this is a very uncommon procedure and most surgeons only performed one to three of these cases throughout the registry time period. This may lend to developing specialized high-volume explant centres as the number of patients requiring this type of surgical procedure increases.

\section{Coronary access when considering redo-TAVR}

The chances of coronary obstruction are very low in both native TAVR at $0.5 \%$ (33), and as previously mentioned at $0.7 \%$ in the redo-TAVR study (28). However, despite being rare, challenges with coronary access (CA) in patients after redo-TAVR is well established and a potentially devastating concern. Whilst complete coronary artery obstruction has a high mortality, a less dramatic but no less important situation is difficulty trying to get selective CA through the second THV frame when indicated for diagnostic or interventional purposes. Difficulties in engaging the coronary artery may be due to the new position of the native aortic leaflets or hindered by the prosthetic leaflets, metallic cells or the commissural post of the THV (34). If the outflow of the first THV is at or near the Sino tubular junction (STJ), the second THV could 'prop' the first THV leaflets up against the STJ and sequester the aortic sinus and completely impede blood flow into the aortic sinus (35). The largest risk factor for causing difficulties with CA is a pre-existing bioprosthetic valve, other important issues include anatomy of the aortic root and coronary heights (Figure 6) (36). With experienced heart teams and preprocedural MDCT and therefore appropriate patient selection, issues with CA should be a largely avoidable complication.

\section{Case examples of THV reintervention}

\section{Case 1}

A ninety-year-old female presented with recurrent heart failure hospitalisations and severe mixed SVD of a $23 \mathrm{~mm}$ Sapien XT THV, implanted six years prior. Pre-procedure trans-thoracic echocardiography (TTE) showed an elevated trans-aortic gradient of $49 \mathrm{mmHg}$ and moderate valvular AR (Figure $7 A, 7 B$ ). Pre-procedural CT demonstrated no HALT, mildly under-expanded Sapien XT prothesis (average diameter $=21.6 \mathrm{~mm}$ ). A trans-femoral redo-TAVR was performed with pre-dilatation using a $23 \mathrm{~mm}$ True balloon followed by a $23 \mathrm{~mm}$ Sapien 3 THV. Post-deployment aortogram demonstrated no PVL and TTE demonstrate a trans-aortic mean gradient of $6 \mathrm{mmHg}$.

\section{Case 2}

A eighty-eight-year old female presented with NYHA class III symptoms and severe stenotic degeneration of a $21 \mathrm{~mm}$ Perimount surgical bioprosthesis implanted twelve years prior. Heart team consensus recommended a redo-TAVR procedure given very high estimated surgical risk (STS $20.4 \%)$. Pre-procedural CT demonstrated high risk of sinus sequestration simulating a $23 \mathrm{~mm}$ THV (Figure 8A), therefore a $20 \mathrm{~mm}$ Sapien 3 was chosen. Immediately after implantation, the patient experienced cardiac standstill and aortogram demonstrated sinus sequestration (Figure $8 B$ ). With urgent Extracorporeal membrane oxygenation support a 'chimney stent' was implanted restoring coronary flow (Figure 8C). She recovered well and was discharged day four after the procedure. As one-year follow-up, she remains active with NYHA class II symptoms, no HF rehospitalization or bleeding events, and satisfactory THV hemodynamic function (trans-aortic MG of $21 \mathrm{mmHg}$ ). MDCT demonstrated a patent left main stem chimney stent (Figure 8D).

\section{Case 3}

A seventy-eight-year-old female presented with acute coronary syndrome six months after TAVR with $26 \mathrm{~mm}$ Evolut R THV (Medtronic Inc., Minneapolis, Minnesota). Pre-TAVR coronary angiogram and post-procedure THV function were normal. Direct engagement of the left main stem was challenging. An urgent cardiac computedtomography scan showed extensive pannus on the THV frame expanding up to the level of the left main stem and revealed the THV commissure post was in front of the left main coronary ostia.

\section{Case 4}

An eighty-five-year-old male presented with severe 


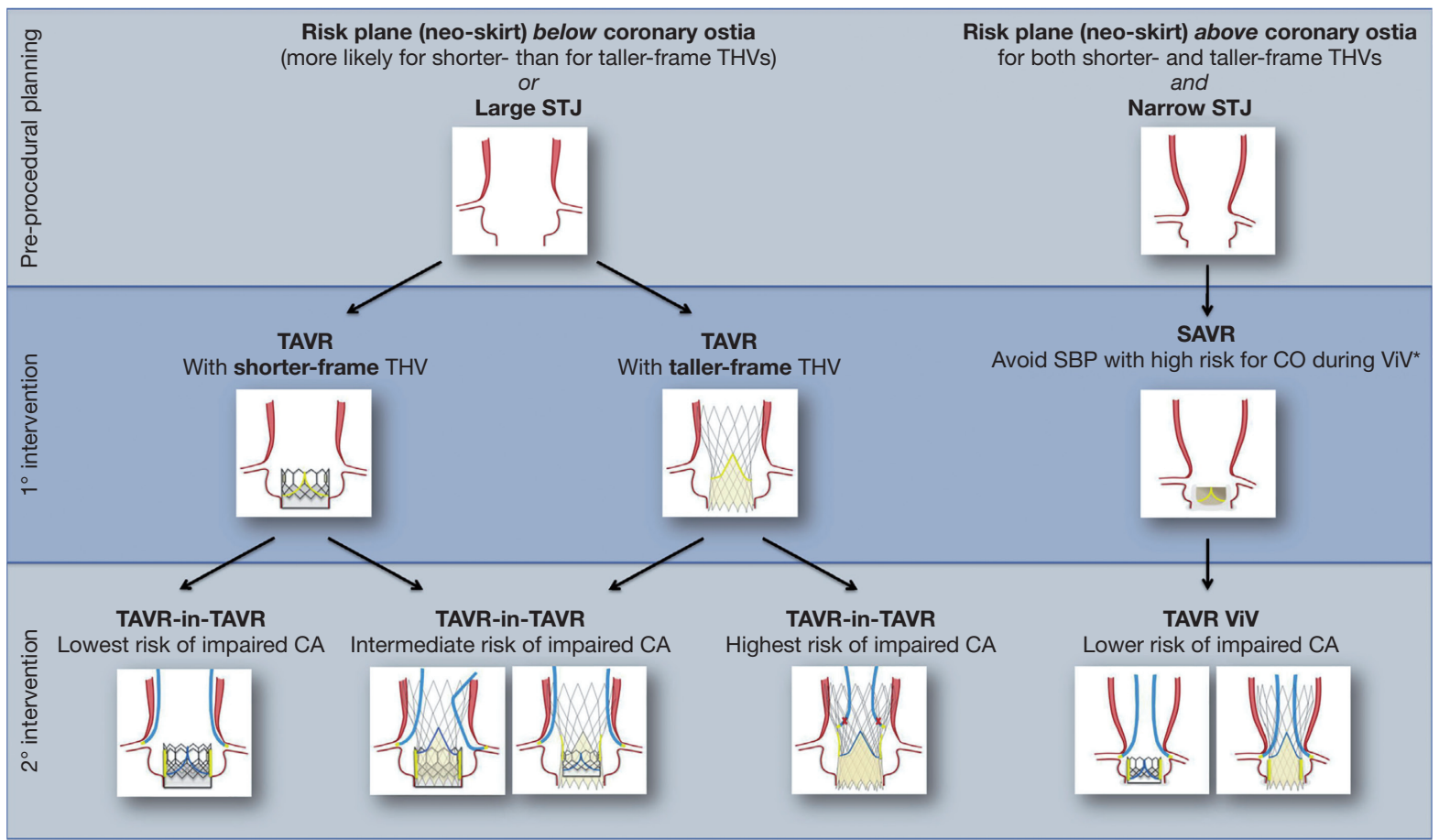

Treatment of severe symptomatic aortic stenosis in younger and lower-risk patients with regard to coronary access after TAVR-in-TAVR. *In particular, stentless surgical bioprostheses and those with externally mounted leaflets. AS = aortic stenosis; $\mathrm{CA}=$ coronary access; $\mathrm{CO}=\mathrm{coronary}$ occlusion; SAVR = surgical aortic valve replacement; SBP = surgical bioprosthesis; STJ = sinotubular junction; TAVR = transcatheter aortic valve replacement; THV = transcatheter heart valve; ViV = valve-in-valve.

Figure 6 Treatment of severe AS in younger/lower risk patients and considerations for coronary access with redo-TAVR (used with permission). AS, aortic stenosis; TAVR, transcatheter aortic valve replacement.
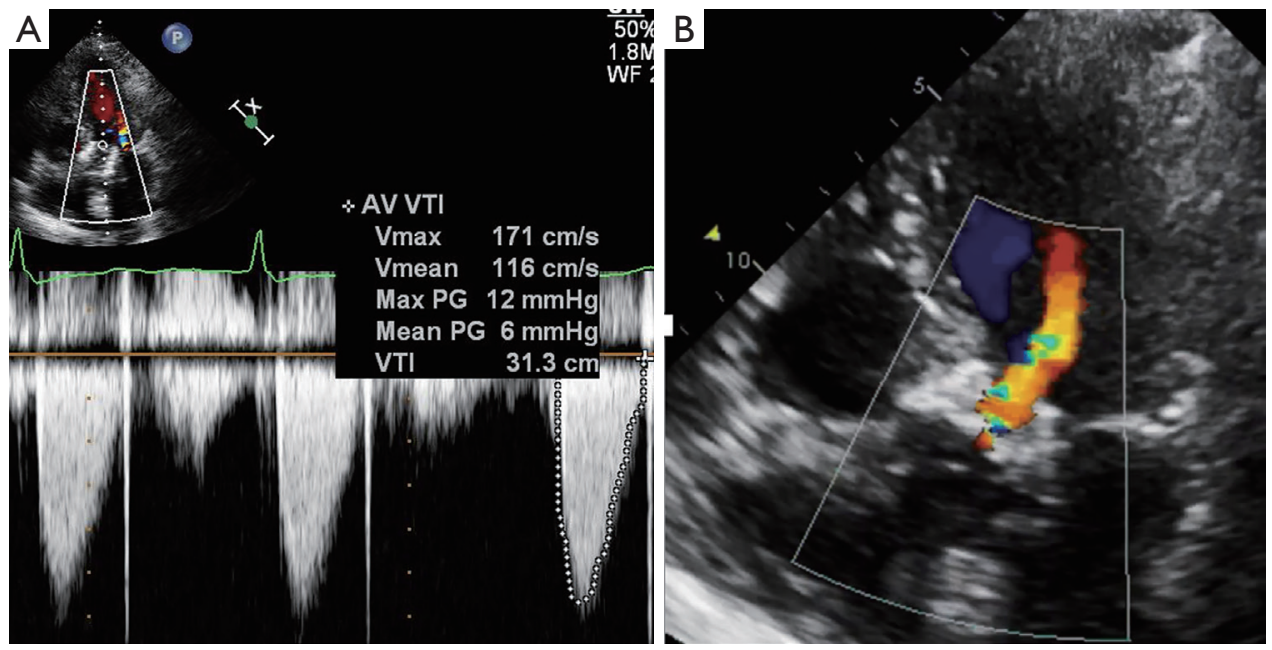

Figure 7 Severely elevated mean gradient across the Sapien XT THV (A). Moderate valvular regurgitation (B). THV, transcatheter heart valve. 

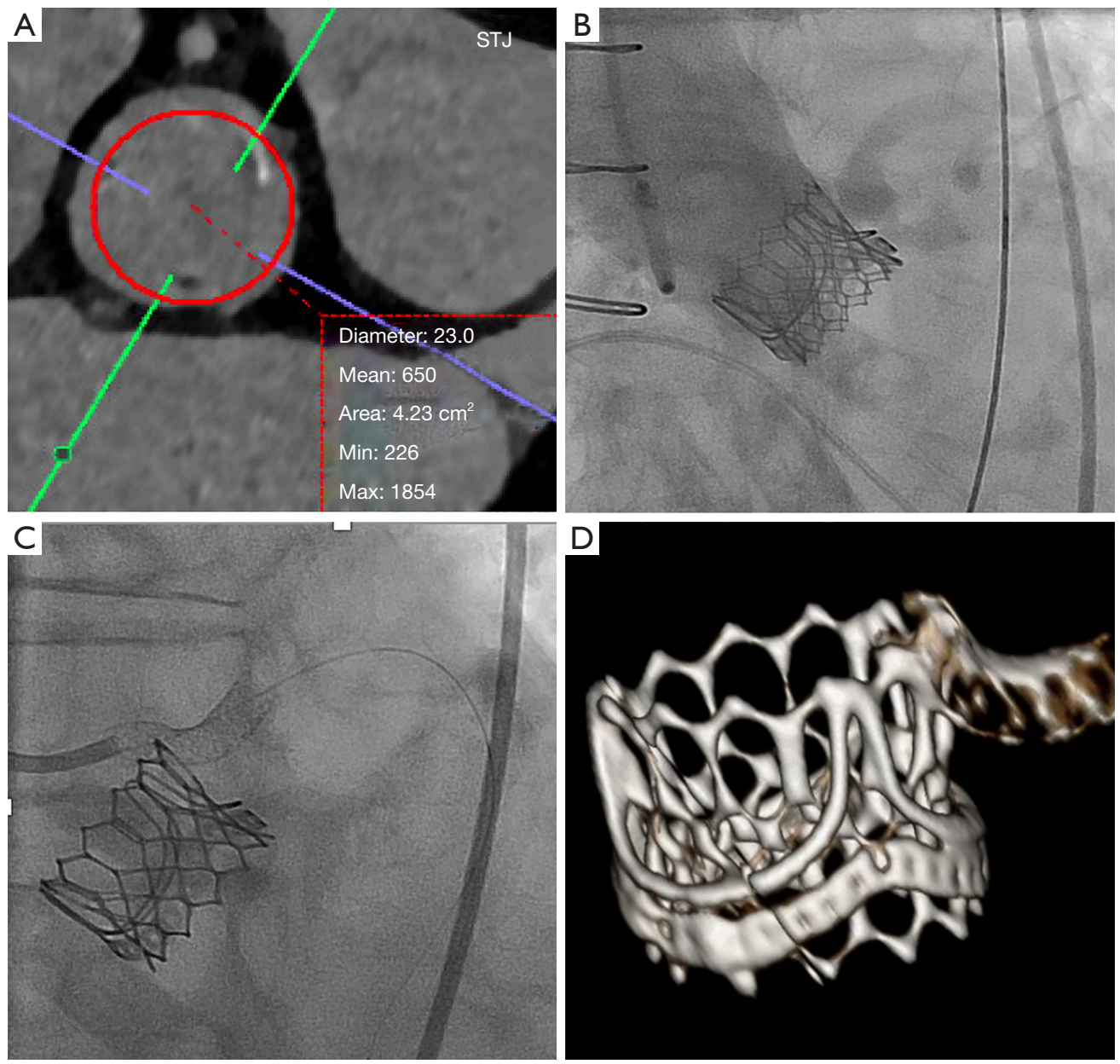

Figure 8 Pre-procedural CT demonstrating risk of sinus sequestration simulating a $23 \mathrm{~mm}$ THV (A). Sinus sequestration after a 20 mm Sapien 3 implantation causing cardiac arrest (B). Chimney stent implantation under extracorporeal membrane oxygenation support (C). Post-procedural CT demonstrates the THV within the surgical valve and a patent chimney stent 30 days post procedure (D). THV, transcatheter heart valve.

regurgitant degeneration of a $27 \mathrm{~mm}$ freestyle aortic bioprosthesis and ascending aortic replacement fifteen years prior. Heart team consensus recommended a ViV procedure with a trans-apical approach given significant angulation at the junction of the previous aortic replacement site. Using trans-apical access with fluoroscopic and TEE guidance, a nominal $26 \mathrm{~mm}$ Sapien 3 THV implanted in the $27 \mathrm{~mm}$ freestyle bioprosthesis. Post-deployment TTE demonstrated well positioned THV with a MG of $12 \mathrm{mmHg}$ at one-month.

\section{Conclusions}

Currently, the future is very exciting for the management of AS, as the field expands with newer THV platforms and the trials demonstrate non-inferior outcomes for younger, lower risk patients. A fundamental component of all successful TAVR programs has been the adoption of the 'heart team' concept which pulls together experts and identifies suitable patients and importantly predicts potential complications. As the use of TAVR expands into lower risk patients the lifetime management of an individual's AS needs to be considered. Two key components in the patient's management need to be considered upfront; the concept of THV durability and the complexities of a redo-TAVR procedure, if required. The treatment of the patient's AS needs to be decided in context of their overall life expectancy, concomitant intervention, the possibility of 
re-intervention and coronary access in the future. Current evidence suggests favourable mid-term outcomes for redoTAVR. Ultimately, despite favourable reports from registry data, larger long-term studies are required to assess the durability and repeatability of redo-TAVR.

\section{Acknowledgments}

Funding: None.

\section{Footnote}

Conflicts of Interest: Dr. AGC is funded by the New Zealand Heart Foundation and John Ormiston Scholarship. Dr. Anson C is consultant to Medtronic. Dr. MA received research grant from Fédération Française de cardiologie, MUSE-Explore and Biotronik. Dr. Anthony C is funded by the Royal Australasian College of Physicians Vincent Fairfax Research Entry Scholarship. Dr. DAW receives unrestricted grant support from Edwards Lifesciences, Abbott, and Medtronic. Dr. JS is a consultant to Edwards Lifesciences and Medtronic. Dr. JW is a consultant to Edwards Lifesciences. The other authors declare no conflicts of interest.

Open Access Statement: This is an Open Access article distributed in accordance with the Creative Commons Attribution-NonCommercial-NoDerivs 4.0 International License (CC BY-NC-ND 4.0), which permits the noncommercial replication and distribution of the article with the strict proviso that no changes or edits are made and the original work is properly cited (including links to both the formal publication through the relevant DOI and the license). See: https://creativecommons.org/licenses/by-nc-nd/4.0/.

\section{References}

1. Leon MB, Smith CR, Mack M, et al. Transcatheter aorticvalve implantation for aortic stenosis in patients who cannot undergo surgery. N Engl J Med 2010;363:1597-607.

2. Smith CR, Leon MB, Mack MJ, et al. Transcatheter versus surgical aortic-valve replacement in high-risk patients. $\mathrm{N}$ Engl J Med 2011;364:2187-98.

3. Adams DH, Popma JJ, Reardon MJ, et al. Transcatheter aortic-valve replacement with a self-expanding prosthesis. N Engl J Med 2014;370:1790-8.

4. Leon MB, Smith CR, Mack MJ, et al. Transcatheter or Surgical Aortic-Valve Replacement in Intermediate-Risk
Patients. N Engl J Med 2016;374:1609-20.

5. Reardon MJ, Van Mieghem NM, Popma JJ, et al. Surgical or Transcatheter Aortic-Valve Replacement in Intermediate-Risk Patients. N Engl J Med 2017;376:1321-31.

6. Popma JJ, Deeb GM, Yakubov SJ, et al. Transcatheter Aortic-Valve Replacement with a Self-Expanding Valve in Low-Risk Patients. N Engl J Med 2019;380:1706-15.

7. Mack MJ, Leon MB, Thourani VH, et al. Transcatheter Aortic-Valve Replacement with a Balloon-Expandable Valve in Low-Risk Patients. N Engl J Med 2019;380:1695-705.

8. Capodanno D, Petronio AS, Prendergast B, et al. Standardized definitions of structural deterioration and valve failure in assessing long-term durability of transcatheter and surgical aortic bioprosthetic valves: a consensus statement from the European Association of Percutaneous Cardiovascular Interventions (EAPCI) endorsed by the European Society of Cardiology (ESC) and the European Association for Cardio-Thoracic Surgery (EACTS). Eur Heart J 2017;38:3382-90.

9. Dvir D, Bourguignon T, Otto CM, et al. Standardized Definition of Structural Valve Degeneration for Surgical and Transcatheter Bioprosthetic Aortic Valves. Circulation 2018;137:388-99.

10. Pibarot P, Dumesnil JG. Prosthetic heart valves: selection of the optimal prosthesis and long-term management. Circulation 2009;119:1034-48.

11. VARC-3 WRITING COMMITTEE, Généreux P, Piazza N, et al. Valve Academic Research Consortium 3: Updated Endpoint Definitions for Aortic Valve Clinical Research. J Am Coll Cardiol 2021;77:2717-46.

12. Rodriguez-Gabella T, Voisine P, Puri R, et al. Aortic Bioprosthetic Valve Durability: Incidence, Mechanisms, Predictors, and Management of Surgical and Transcatheter Valve Degeneration. J Am Coll Cardiol 2017;70:1013-28.

13. Otto CM, Nishimura RA, Bonow RO, et al. 2020 ACC/ AHA Guideline for the Management of Patients With Valvular Heart Disease: Executive Summary: A Report of the American College of Cardiology/American Heart Association Joint Committee on Clinical Practice Guidelines. Circulation 2021;143:e35-71.

14. Kodali SK, Williams MR, Smith CR, et al. Two-year outcomes after transcatheter or surgical aortic-valve replacement. N Engl J Med 2012;366:1686-95.

15. Tamburino C, Capodanno D, Ramondo A, et al. Incidence and predictors of early and late mortality after transcatheter aortic valve implantation in 663 patients with 
severe aortic stenosis. Circulation 2011;123:299-308.

16. Leetmaa T, Hansson NC, Leipsic J, et al. Early aortic transcatheter heart valve thrombosis: diagnostic value of contrast-enhanced multidetector computed tomography. Circ Cardiovasc Interv 2015;8:e01596.

17. Pache G, Schoechlin S, Blanke P, et al. Early hypoattenuated leaflet thickening in balloon-expandable transcatheter aortic heart valves. Eur Heart J 2016;37:2263-71.

18. Rodriguez-Gabella T, Voisine P, Dagenais F, et al. LongTerm Outcomes Following Surgical Aortic Bioprosthesis Implantation. J Am Coll Cardiol 2018;71:1401-12.

19. Cribier A, Eltchaninoff $H$, Bash A, et al. Percutaneous transcatheter implantation of an aortic valve prosthesis for calcific aortic stenosis: first human case description. Circulation 2002;106:3006-8.

20. Daubert MA, Weissman NJ, Hahn RT, et al. Long-Term Valve Performance of TAVR and SAVR: A Report From the PARTNER I Trial. JACC Cardiovasc Imaging 2016. [Epub ahead of print]. doi: 10.1016/j.jcmg.2016.11.004.

21. Barbanti M, Petronio AS, Ettori F, et al. 5-Year Outcomes After Transcatheter Aortic Valve Implantation With CoreValve Prosthesis. JACC Cardiovasc Interv 2015;8:1084-91.

22. Søndergaard L, Ihlemann N, Capodanno D, et al. Durability of Transcatheter and Surgical Bioprosthetic Aortic Valves in Patients at Lower Surgical Risk. J Am Coll Cardiol 2019;73:546-53.

23. Didier R, Eltchaninoff H, Donzeau-Gouge P, et al. Five-Year Clinical Outcome and Valve Durability After Transcatheter Aortic Valve Replacement in High-Risk Patients. Circulation 2018;138:2597-607.

24. Webb JG, Mack MJ, White JM, et al. Transcatheter Aortic Valve Implantation Within Degenerated Aortic Surgical Bioprostheses: PARTNER 2 Valve-in-Valve Registry. J Am Coll Cardiol 2017;69:2253-62.

25. Deeb GM, Chetcuti SJ, Reardon MJ, et al. 1-Year Results in Patients Undergoing Transcatheter Aortic Valve Replacement With Failed Surgical Bioprostheses. JACC Cardiovasc Interv 2017;10:1034-44.

26. Deharo P, Bisson A, Herbert J, et al. Transcatheter Valvein-Valve Aortic Valve Replacement as an Alternative to Surgical Re-Replacement. J Am Coll Cardiol 2020;76:489-99.

27. Al-Abcha A, Saleh Y, Boumegouas M, et al. Meta-Analysis of Valve-in-Valve Transcatheter Aortic Valve Implantation
Versus Redo-surgical Aortic Valve Replacement in Failed Bioprosthetic Aortic Valve. Am J Cardiol 2021;146:74-81.

28. Landes U, Webb JG, De Backer O, et al. Repeat

Transcatheter Aortic Valve Replacement for Transcatheter Prosthesis Dysfunction. J Am Coll Cardiol 2020;75:1882-93.

29. Sathananthan J, Hensey M, Landes U, et al. Long-Term Durability of Transcatheter Heart Valves: Insights From Bench Testing to 25 Years. JACC Cardiovasc Interv 2020;13:235-49.

30. Sathananthan J, Fraser R, Landes U, et al. Repeat transcatheter aortic valve replacement and implications for transcatheter heart valve performance: insights from bench testing. EuroIntervention 2021. [Epub ahead of print]. doi: 10.4244/EIJ-D-20-00697.

31. Pasala TKR, Ruiz CE. Transcatheter Aortic Valve Replacement for All-comers With Severe Aortic Stenosis: Could It Become a Reality? Rev Esp Cardiol (Engl Ed) 2018;71:141-5.

32. Fukuhara S, Brescia AA, Deeb GM. Surgical Explantation of Transcatheter Aortic Bioprostheses: An Analysis From the Society of Thoracic Surgeons Database. Circulation 2020;142:2285-7.

33. Holmes DR Jr, Nishimura RA, Grover FL, et al. Annual Outcomes With Transcatheter Valve Therapy: From the STS/ACC TVT Registry. J Am Coll Cardiol 2015;66:2813-23.

34. Nai Fovino L, Scotti A, Massussi M, et al. Incidence and feasibility of coronary access after transcatheter aortic valve replacement. Catheter Cardiovasc Interv 2020;96:E535-41.

35. Tang GHL, Zaid S, Gupta E, et al. Feasibility of Repeat TAVR After SAPIEN 3 TAVR: A Novel Classification Scheme and Pilot Angiographic Study. JACC Cardiovasc Interv 2019;12:1290-2.

36. Tarantini G, Nai Fovino L. Coronary Access and TAVRin-TAVR: Don't Put Off Until Tomorrow What You Can Do Today. JACC Cardiovasc Interv 2020;13:2539-41.

Cite this article as: Chatfield AG, Cheung A, Akodad M, Chuang A, Besola L, Sellers S, Wood DA, Sathananthan J, Webb J. Transcatheter solutions for transcatheter aortic valve replacement dysfunction: is redo transcatheter aortic valve replacement a durable option? Ann Cardiothorac Surg 2021;10(5):571-584. doi: 10.21037/acs-2021-tviv-85 